\title{
Insect-inspired vision for autonomous vehicles
}

\author{
Julien R. Serres ${ }^{1}$ and Stéphane Viollet ${ }^{1}$ \\ ${ }^{1}$ Aix Marseille University, CNRS, ISM, Marseille, France
}

September 10, 2018

\section{Highlights:}

- Compound eyes are an endless source of inspiration for developing visual sensors

- Visual stabilization of robot's flight attitude controlled by artificial ocelli

- Ultraviolet celestial cue-based navigation works efficiently under all weather conditions

- Combining blurry vision with retinal micro-movements makes robots' visual tracking hyperacute

\begin{abstract}
Flying insects are being studied these days as if they were agile micro air vehicles fitted with smart sensors, requiring very few brain resources. The findings obtained on these natural fliers have proved to be extremely valuable when it comes to designing compact low-weight artificial optical sensors capable of performing visual processing tasks robustly under various environmental conditions (light, clouds, contrast). Here we review some outstanding bio-inspired visual sensors, which can be used for either detecting motion in the visible spectrum or controlling celestial navigation in the ultraviolet spectrum and for attitude stabilisation purposes. Biologically inspired visual sensors do not have to comprise a very large number of pixels: they are able to perform both short and long range navigation tasks surprisingly well with just a few pixels and a weak resolution.
\end{abstract}

Keywords: Bio-inspired sensors; Biomimicry; Bionics; Biorobotics; Bioinspired robotics. 


\section{Introduction}

Insects are definitely an endless source of inspiration for scientists designing innovative sensors. Findings on the nocturnal butterfly's compound eye with its superimposed optics [3] have been used, for example, to design gradedindex (GRIN) lenses. Studies on the halteres forming the fly's rate gyros 29| have inspired what was probably the first micro rate gyroscope based on a vibrating part, which was subjected to the Coriolis force in order to measure the angular speed [13]. New photonic structures for energy-saving and healthcare applications have recently been developed based on studies on the black butterfly (Pachliopta aristolochiae) [51]. Arthropods' and insects' visual systems have also inspired dedicated visual sensors and small, highly efficient optical sensors with which to equip aerial robots [22]. In this review, it is proposed to describe some insect-inspired visual sensors which have been used in robotic applications, especially for performing autonomous navigation and flight stabilization tasks. Although we focus here on insects' vision, it is worth noting that many other studies [1, 44], such as those on mantis shrimps in particular [11, 63], have led to the production of some outstandingly efficient optical sensors.

\section{Smart bio-inspired sensors}

\section{Artificial compound eyes and motion sensors}

In the field of artificial optical design, since the lens diameter is usually much larger than the light wavelength, the light diffraction is not taken into consideration. The inter pixel angle, defined as $\Delta \phi=\frac{d}{f}$, where $d$ is the pixels' pitch and $f$ is the focal length, and $\Delta \rho$ is the acceptance angle given by the width of the Gaussian angular sensitivity at half height $(\Delta \rho \approx \Delta \phi)$, can be obtained by slightly defocusing the lens. Two artificial compound eyes have been developed, using either inorganic semiconductor photoreceptors comprising 630 pixels, where $\Delta \phi=\Delta \rho=4.2^{\circ}$ [21], mimicking the Drosophila melanogaster's compound eye (Fig. 17), or organic photodiodes comprising 256 pixels, where $\Delta \phi=11^{\circ}$ and $\Delta \rho=9.7^{\circ}[52]$, mimicking the fire ant's Solenopsis fugax compound eye (Fig. 1 $1 \mathrm{~b}$ ).

The CurvACE artificial compound eye was developed in the framework of a European project (2009-2013; www.curvace.org, [21]).This functional prototype with its 630 pixels (forming 630 artificial ommatidia) gave a wide field of view $\left(180^{\circ} \times 60^{\circ}\right)$ in a large range of lighting conditions and weights $\sim 2$-gram 21]. A pair of CurvACE sensors was mounted on board an 80- 
gram micro flying robot called BeeRotor [19] for performing both ground and ceiling avoidance tasks. A minimalistic version of this artificial compound eye containing only three photoreceptors set under a common lens, weighing only $2 \mathrm{mg}$ and detecting movements in two dimensions at rates of up to 300 $\mathrm{Hz}$ with an ultra low current consumption of only $0.444 \mathrm{~mA}$, has also been constructed [43] (Fig. 2d).

A recent optic flow sensor based on the $\mathrm{M}^{2} \mathrm{APix}$ retina $\left(\mathrm{M}^{2} \mathrm{APix}\right.$ stands for Michaelis-Menten Auto-adaptive Pixel [36], Fig. 16) can auto-adapt in a 7 -decade lighting range and responds appropriately to step changes of up to \pm 3 decades [37,56]. These pixels do not saturate thanks to the normalization process performed by Very Large Scale Integration (VLSI) transistors [36]; this advantage is due to the intrinsic properties of the Michaelis-Menten equation [40] on which these pixels are based. Comparisons between the characteristics of auto-adaptive Michaelis-Menten and Delbrück pixels [12] under identical lighting conditions (i.e., with the pixels integrated into the same retina) showed that the Michaelis-Menten pixels gave better performances in terms of their dynamic sensitivity and minimum contrast detection levels [36].

Algorithms of several kinds have been developed for computing local motion, which have resulted in various hardware implementations including templates, time-of-travel, feature tracking, edge counting, edge correlation devices and the Hassenstein-Reichardt correlator [5, 39, 62], as well as some software implementations [5, 56]. However, analog VLSI motion sensors significantly reduce micro flying robots' power consumption and payload while increasing the bandwidth, thus improving both the precision and the accuracy of the onboard optic flow measurements.

\section{Artificial ocelli}

Ocelli are visual sensors which are present in the dorsal part of many insects's heads (Fig. 2a). The word ocellus means a little eye. One to three ocelli can be found, at different points on the top of insects' heads [31]. Ocelli usually consist of three elementary eyes having a lens with a relatively large diameter (ranging from $100 \mu \mathrm{m}$ to $500 \mu \mathrm{m}$ ) and a small focal length. Because of the large aperture and the resulting f-number $N$ of the lens $\left(N=\frac{f}{D}\right.$, where $f$ is the focal length and $D$ is the diameter of the aperture), ocelli can detect low light levels, but the lens is positioned in such a way that the image projected onto the photoreceptors is always under-focused. The exact function of the ocelli has not yet been elucidated, but behavioural experiments on flies have shown that the ocelli are involved in head stabilisation processes [28, 48], and electrophisiological recordings on dragonflies have even shown that the second-order neuron (L-neuron) directly connected to the 

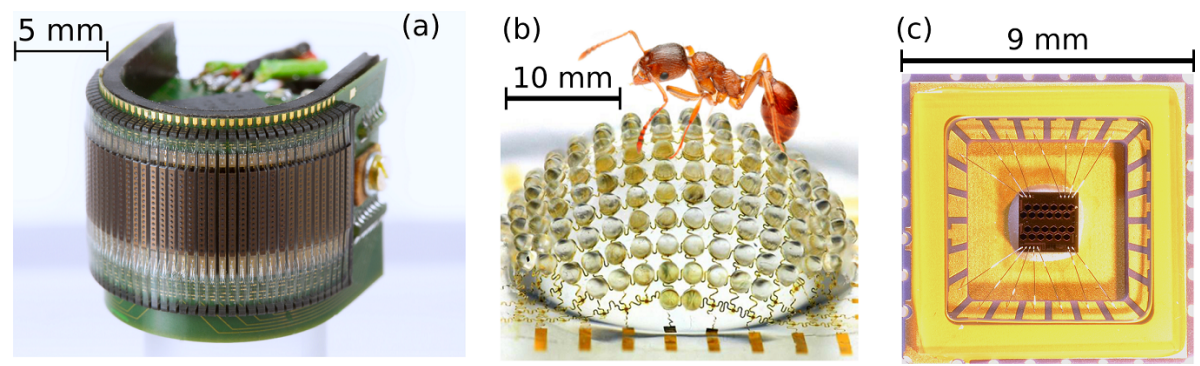

Figure 1: (a) The first artificial compound eye constructed in the framework of the CurvACE European project (www.curvace.org) [21]. Photographic credit: Floreano Dario. This 2-gram sensor includes a matrix of auto-adaptive pixels (630 Delbrück pixels), each of which is covered by a micro lens forming a juxtaposed set of small cylindrically curved elementary eyes, which are comparable in number to the eyes of the fruit fly Drosophila.(b) The first 256-pixel artificial compound eye, which is a moulded piece of poly(dimethylsiloxane) (PDMS) elastomer carrying an array of 16 $\mathrm{x} 16$ convex microlenses covering an area of $14.72 \mathrm{~mm} \times 14.72 \mathrm{~mm} \mathrm{[52]:} \mathrm{this}$ number is similar to the number of microlenses present in the eyes of the fire ant Solenopsis fugax. Courtesy from John Roger, University of Illinois and Beckman Institute. (c) The $\mathrm{M}^{2} \mathrm{APix}$ optic flow sensor is composed of 12 Michaelis-Menten pixels (top) and 12 Delbrück pixels (bottom). Adapted from 36$]$.

photoreceptors may be sensitive to preferred motion in ultraviolet light [2]. In short, the ocelli are closely involved in the stabilization reflexes responsible for controlling the fly's body rotation (on the pitch and roll axes) and the head orientation (in gaze control processes). Ocellar signals are fused with compound eye signals [42] but the ocelli are faster than the compound eyes because a much smaller number of neural processing layers are involved in the processing of the ocellar signals transmitted to the descending neurons [55]. On the basis of results obtained in biological studies, several artificial ocelli have been developed and tested. Chahl and Mizutani (2012) developed a biomimetic eye composed of 4 pairs of photodiodes which were sensitive to ultraviolet and green lights [4] (Fig. 2c). Artificial ocelli were used by the latter authors to stabilize the roll movements of a small unmanned aerial (fixed wing) vehicle [4]. Gremillion et al. (2012-2014) developed an analog ocellar sensor composed of four photodiodes with a response range of 350-1050 nm, which was used to estimate the pitch and roll movements of an aerial robotic platform 24, 25] (Fig. 2b). Fuller et al. presented a method of stabilizing a micro flying insect by means of an ocelli-inspired sensor (Fig. $2 \mathrm{~b}$ ) to ob- 
tain angular velocity feedback (instead of attitude feedback) and used this method to stabilize an insect-scale robot in short take-off flights [23].
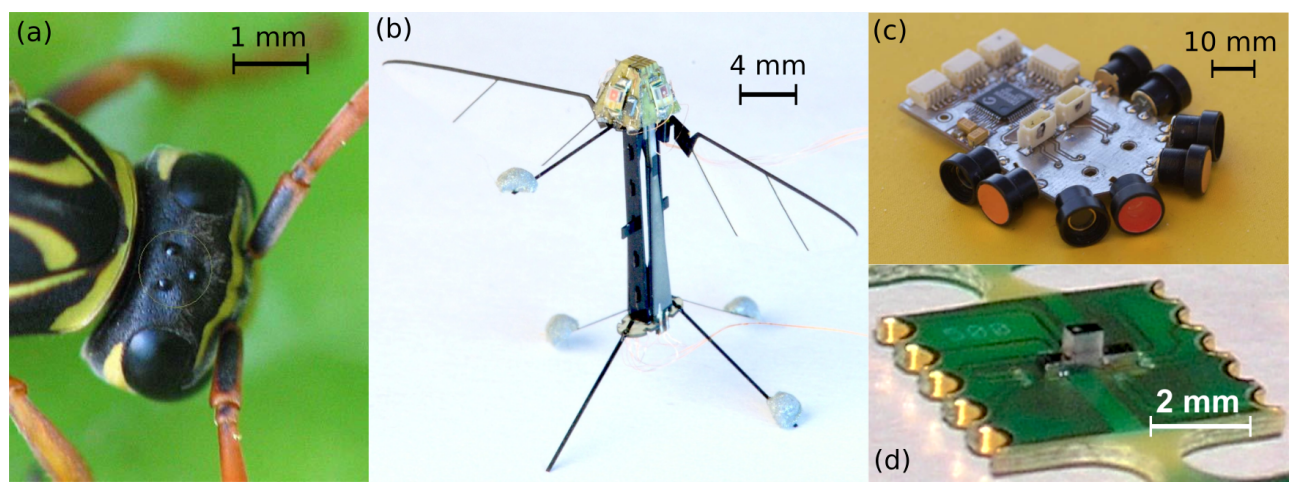

Figure 2: (a) Top view of the wasp's head Poliste from Wikimedia commons (Photographic credit Assafn (2008) under license CC-BY-SA 3.0). Ocelli (a group of 3 small eyes) forming a triangle on the dorsal part of the Poliste bee's head (Poliste's head width: 3.6mm). (b) Picture of a 25-mg ocelliinspired sensor with four phototransistors arranged in a pyramid at the top of an insect-like flying robot called Robobee. Courtesy from Swayer B. Fuller, University of Washington, Seattle, USA. See 23 for details. (c) Artificial ocelli composed of 8 photodiodes (four ultraviolet/green pairs). The sensor identifies the horizon based on the polarization of the sky. Adapted from [4]. Photographic credits: Javaan Chahl and Akiko Mizutani. (d) Picture of a photoreceptor triplet mounted on the PCB surface. Adapted from [43]. Photographic credits: Ramon Pericet-Camara and Floreano Dario, Laboratory of Intelligent Systems, EPFL.

\section{Celestial compass inspired by insects'dorsal rim area}

To be able to travel long distances, insects have to keep a constant heading relative to a stable reference point [60]. Desert ants (Cataglyphis fortis) are able to home with a high level of accuracy, based on their knowledge of their heading acquired when exploring the environment [60]. Locusts [38], flies [59, 61, bees [45, 46], ants and dung beetles [10] are equipped with an outstandingly efficient optical compass, which is sensitive to the polarized light caused by the atmospheric scattering of the sunlight. The scattering of the sunlight within the Earth's atmosphere produces a polarization pattern across the sky. Solar radiations remain unpolarized until their entry into the atmosphere, where scattering interactions with atmospheric constituents induce the partially linear polarization of the skylight [9]. Insects possess 
photoreceptors in the dorsal region of their eye (the dorsal rim area, DRA) that are specialized in detecting the pattern of polarized skylight $32,60,61$. The first robotic application of the desert ants' DRA was presented in |34 (Fig. 3a). Chu et al. developed a smaller compass consisting of 6 photodiodes topped by linear polarizers set $60^{\circ}$ apart $[6]$. A pair of polarized-light sensors of this kind were tested under a clear sky (Fig. 3b): the accuracy of these polarized-light sensors was found to be within $\pm 0.2^{\circ}$ [58|. A version of the polarization sensor shown in Fig. 3b, composed of just a single unit, which was recently mounted on board a quadrotor (Fig. $3 \mathrm{~d}$ ), gave promising performances: indoor accuracy $0.2^{\circ}$, outdoor accuracy less than $2^{\circ}$, and output refresh signal $10 \mathrm{~Hz}$ [64]. A polarization-based photodetector involving a bilayer nanowire was recently developed and tested under artificial blue lighting, giving a mean error of only $\pm 0.1^{\circ}$ once a polynomial fitting process had been applied [7]. Other applications of wire grid micro-polarizers have been developed, some of which involve various angles of polarization (in regular steps of $45^{\circ}$ ) [30]. Bio-inspired approaches have also been adopted with cameras in the visible range [20] along with the polarization model developed in [35. The brand new celestial sensor recently developed by the French Biorobotics group in Marseille is composed of two polarization units (POLunits) consisting of only two UV light sensors topped with actuated rotating linear sheet polarizers (Fig. 3c). This approach makes it possible to greatly reduce the number of pixels, but at the expense of the temporal resolution, which is limited by the mechanical rotational speed. This sensor mimicks the UV-sensitivity of the ant's photoreceptors and the log ratio of two orthogonal POL-units, as previously proposed in the Labhart's locust-based model [33]. In addition, this insect-based compass, which is highly reliable and suitable for performing navigation tasks in various meteorological contexts [15], is able to determine its heading angle with great precision: the median error recorded was only $2.9^{\circ}$ when the sky was slightly cloudy, and $1.9^{\circ}$ in the case of an overcast sky [16]. Several artificial celestial compass sensors have been produced for robotic and autonomous navigation purposes [17, 34, 35, 64].

\section{Fly-inspired hyperacute sensor}

The accuracy (acuity) of a contrasting object's angular position measurements depends directly on the position sensor's optical resolution. The acuity of a compound eye might therefore be expected to be relatively poor in comparison with that of the human camerular eye, which contains several million photoreceptors (i.e., pixels). This would be true without the existence of the fly's active visual processing system based on retinal micro vibrations. Since the 1970s, several studies have shown that active retinal micro movements 

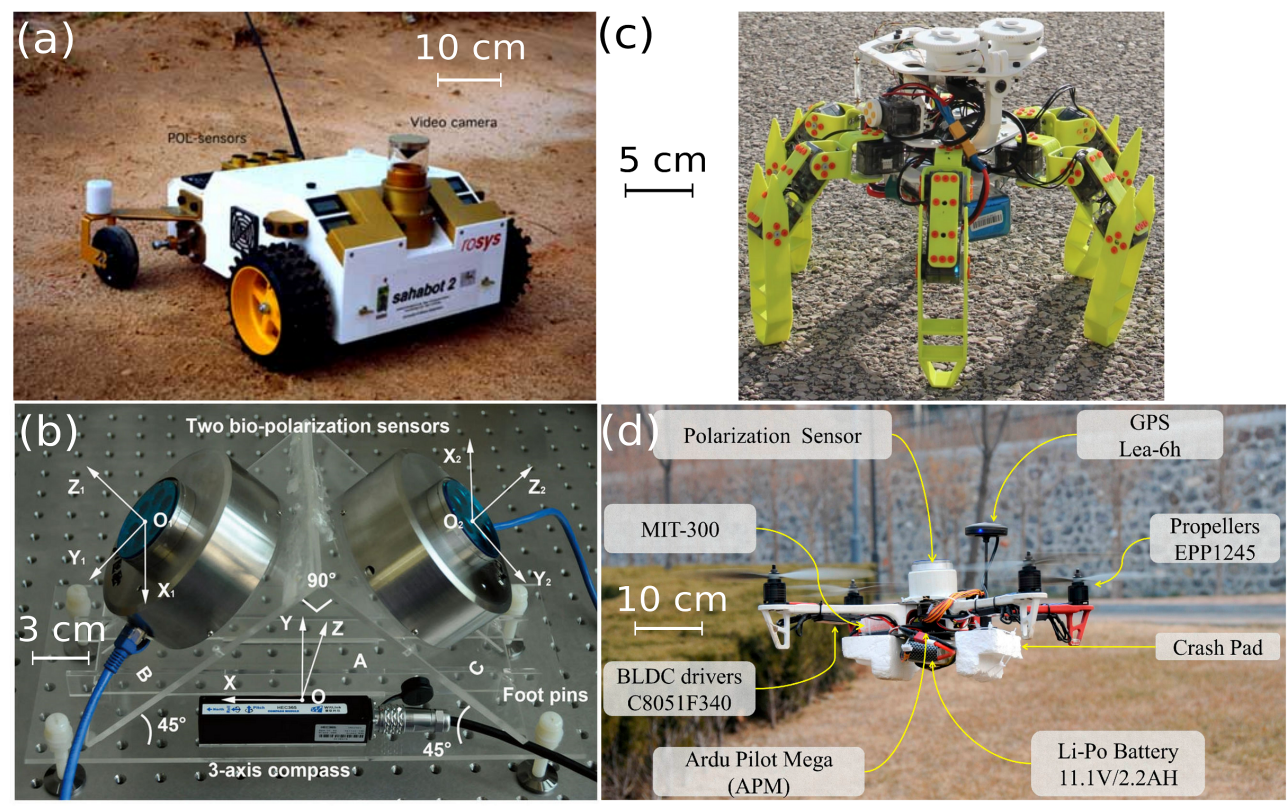

Figure 3: (a) The Sahabot 2 robot (2000) with its ant-inspired compass from [35 by permission of Elsevier. (b) Device based on two polarization sensors measuring the heading from [58] under CC-BY License, 2015. (c) Hexabot robot equipped with a pair of UV-polarized light sensors forming a celestial compass from [15]. Photographic credits: Julien Dupeyroux, The Institute of Movement Sciences, CNRS/Aix Marseille University, 2017. (d) Implementation of an extended Kalman filter on board a quadrotor for incorporating the polarization sensor into a conventional attitude determination system, from [64] under CC-BY license, 2018.

occur in flies, but the exact function of these micro movements and how they contribute to improving the visual acuity have not yet been established [57]. However, several authors have integrated micro movements of this kind into their artificial visual sensors, and the results obtained have shown that they were able to locate a contrasting target with much greater accuracy (up to 700-fold) than that achieved using optical systems alone [22, 57]. Thanks to this hyperacuity, it was recently established using a biorobotic approach that a contrasting moving target (moving hands) could be located on a textured background and the distance travelled by a micro robot flying above a textured plane could be measured by processing the visual signals conveyed by the 40 ommatidia in a vibrating artificial compound eye [8]. The presence of hyperacuity has not yet been clearly determined in insects, but a highly counter-intuitive principle in which coarse and blurry vision (due to 
the Gaussian angular sensitivities of the photoreceptors) is combined with vibration seems to be able to give a more accurate perception of the world.

\section{Insect-based visual guidance}

Several ethological experiments have suggested that flying insects rely heavily on optic flow to avoid obstacles and directly control their flight speed in the presence of wind disturbances or changes in the configuration of the environment, as well as to achieve a smooth landing [18, 49,53]. The magnitude of the translational optic flow component is inversely proportional to the distance, which enables flying insects to spontaneously sense the environmental configuration. Two main models have been developed to explain how insects detect the optic flow: the Hassenstein-Reichardt correlator 26 and the time-of-travel model [22]. Although the optic flow is not only used by flying insects [47, 49], it has been frequently used in biologically inspired robots to equip them with insect-inspired vision for both short and long range navigation purposes $449,53,54$.

\section{Conclusion}

The latest advances in the field of insect-like robots will enable roboticists to test biological hypotheses on the scale of insects in order to check whether their hypotheses hold true in the physical world [14, 23, 27, 41, 50]. The expanding set of roles for which robots have been designed means that they will be able to operate in the near future in many contexts such as cluttered and confined environments including buildings, warehouses, industrial plants, performance halls, urban canyons and forests. In these complex environments, autonomous vehicles will require redundant sensors to improve the reliability of their autopilots. The data transmitted by bio-inspired sensors could therefore usefully complement the existing measurements provided by classical sensors such as global positioning systems and magnetometers.

\section{Acknowledgements}

The authors thank J. Diperi and M. Boyron for their technical support and J. Blanc for improving the English manuscript. This research was supported by the French Defense Agency (DGA), CNRS, Aix-Marseille University, Provence-Alpes-Côte d'Azur région and the French National Research Agency (ANR) via the IRIS and Equipex/ Robotex projects. 


\section{References}

[1] Friedrich G Barth, Joseph AC Humphrey, and Mandyam V Srinivasan. Frontiers in sensing: from biology to engineering. Springer Science \& Business Media, 2012.

[2] Richard Berry, Joshua Van Kleef, and Gert Stange. The mapping of visual space by dragonfly lateral ocelli. Journal of Comparative Physiology A, 193(5):495-513, 2007.

[3] Alexander Borst. Drosophila's view on insect vision. Current biology, 19(1):R36-R47, 2009.

[4] Javaan Chahl and Akiko Mizutani. Biomimetic attitude and orientation sensors. IEEE Sensors Journal, 12(2):289-297, 2012.

[5] Haiyang Chao, $\mathrm{Yu} \mathrm{Gu}$, and Marcello Napolitano. A survey of optical flow techniques for robotics navigation applications. Journal of Intelligent 86 Robotic Systems, 73(1-4):361, 2014.

[6] Jinkui Chu, Kaichun Zhao, Qiang Zhang, and Tichang Wang. Construction and performance test of a novel polarization sensor for navigation. Sensors and Actuators A: Physical, 148(1):75-82, 2008.

[7] JK Chu, ZW Wang, Le Guan, Ze Liu, YL Wang, and Ran Zhang. Integrated polarization dependent photodetector and its application for polarization navigation. IEEE Photonics Technol. Lett, 26(5):469-472, 2014 .

[8] Fabien Colonnier, Augustin Manecy, Raphaël Juston, Hanspeter Mallot, Robert Leitel, Dario Floreano, and Stéphane Viollet. A small-scale hyperacute compound eye featuring active eye tremor: application to visual stabilization, target tracking, and short-range odometry. Bioinspiration $\mathscr{E}$ biomimetics, 10(2):026002, 2015.

[9] Kinsell L Coulson. Polarization and Intensity of Light in the Atmosphere. A Deepak Pub, 1988.

[10] Marie Dacke, Dan-Eric Nilsson, Clarke H Scholtz, Marcus Byrne, and Eric J Warrant. Animal behaviour: insect orientation to polarized moonlight. Nature, 424(6944):33, 2003. 
[11] Ilse M Daly, Martin J How, Julian C Partridge, Shelby E Temple, N Justin Marshall, Thomas W Cronin, and Nicholas W Roberts. Dynamic polarization vision in mantis shrimps. Nature communications, 7:12140, 2016.

[12] Tobi Delbruck and Carver A Mead. Adaptive photoreceptor with wide dynamic range. In Circuits and Systems, 1994. ISCAS'94., 1994 IEEE International Symposium on, volume 4, pages 339-342. IEEE, 1994.

[13] H. Droogendijk, R. A. Brookhuis, M. J. de Boer, R. G. P. Sanders, and G. J. M. Krijnen. Towards a biomimetic gyroscope inspired by the fly's haltere using microelectromechanical systems technology. Journal of The Royal Society Interface, 11(99), 2014.

[14] Pierre-Emile J Duhamel, Néstor O Pérez-Arancibia, Geoffrey L Barrows, and Robert J Wood. Biologically inspired optical-flow sensing for altitude control of flapping-wing microrobots. IEEE/ASME Transactions on Mechatronics, 18(2):556-568, 2013.

[15] Julien Dupeyroux, Julien Diperi, Marc Boyron, Stéphane Viollet, and Julien Serres. A bio-inspired celestial compass applied to an ant-inspired robot for autonomous navigation. In ECMR-European Conference on Mobile Robotics, 2017.

[16] Julien Dupeyroux, Julien Diperi, Marc Boyron, Stéphane Viollet, and Julien Serres. A bio-inspired celestial compass for a hexapod walking robot in outdoor environment. In IEEE/RSJ International Conference on Intelligent Robots and Systems (IROS 2017), 2017.

- First robust ant-inspired celestial compass composed of only two pixels.

[17] Julien Dupeyroux, Julien Serres, and Stéphane Viollet. A hexapod walking robot mimicking navigation strategies of desert ants cataglyphis. In Conference on Biomimetic and Biohybrid Systems, pages 145-156. Springer, 2018.

[18] Martin Egelhaaf, Roland Kern, and Jens Peter Lindemann. Motion as a source of environmental information: a fresh view on biological motion computation by insect brains. Frontiers in neural circuits, 8:127, 2014.

[19] Fabien Expert and Franck Ruffier. Flying over uneven moving terrain based on optic-flow cues without any need for reference frames or accelerometers. Bioinspiration \& biomimetics, 10(2):026003, 2015. 
[20] Chen Fan, Xiaoping Hu, Junxiang Lian, Lilian Zhang, and Xiaofeng He. Design and calibration of a novel camera-based bio-inspired polarization navigation sensor. IEEE Sensors Journal, 16(10):3640-3648, 2016.

[21] Dario Floreano, Ramon Pericet-Camara, Stéphane Viollet, Franck Ruffier, Andreas Brückner, Robert Leitel, Wolfgang Buss, Mohsine Menouni, Fabien Expert, Raphaël Juston, et al. Miniature curved artificial compound eyes. Proceedings of the National Academy of Sciences, 110(23):9267-9272, 2013.

•• First functional artificial compound eye for robotic applications.

[22] Nicolas Franceschini. Small brains, smart machines: from fly vision to robot vision and back again. Proceedings of the IEEE, 102(5):751-781, 2014 .

[23] Sawyer B Fuller, Michael Karpelson, Andrea Censi, Kevin Y Ma, and Robert J Wood. Controlling free flight of a robotic fly using an onboard vision sensor inspired by insect ocelli. Journal of The Royal Society Interface, 11(97):20140281, 2014.

-• First insect-scale flying robot fitted with an artificial ocelli.

[24] Gregory Gremillion, Marissa Galfond, Holger G Krapp, and J Sean Humbert. Biomimetic sensing and modeling of the ocelli visual system of flying insects. In Intelligent Robots and Systems (IROS), 2012 IEEE/RSJ International Conference on, pages 1454-1459. IEEE, 2012.

[25] Gregory Gremillion, J Sean Humbert, and Holger G Krapp. Bio-inspired modeling and implementation of the ocelli visual system of flying insects. Biological cybernetics, 108(6):735-746, 2014.

[26] Bernhard Hassenstein and Werner Reichardt. Systemtheoretische analyse der zeit-, reihenfolgen-und vorzeichenauswertung bei der bewegungsperzeption des rüsselkäfers chlorophanus. Zeitschrift für Naturforschung B, 11(9-10):513-524, 1956.

[27] E Farrell Helbling, Sawyer B Fuller, and Robert J Wood. Altitude estimation and control of an insect-scale robot with an onboard proximity sensor. In Robotics Research, pages 57-69. Springer, 2018.

[28] Roland Hengstenberg. Multisensory control in insect oculomotor systems. Visual Motion and its Role in the Stabilization of Gaze, 5:285-298, 1993. 
[29] Roland Hengstenberg. Biological sensors: Controlling the fly's gyroscopes. Nature, 392(6678):757, 1998.

[30] Salmah B Karman, S Zaleha M Diah, and Ille C Gebeshuber. Bioinspired polarized skylight-based navigation sensors: A review. Sensors, 12(11):14232-14261, 2012.

[31] Holger G. Krapp. Ocelli. Current Biology, 19(11):R435-R437, June 2009.

[32] Thomas Labhart. Specialized photoreceptors at the dorsal rim of the honeybee's compound eye: polarizational and angular sensitivity. Journal of comparative physiology, 141(1):19-30, 1980.

[33] Thomas Labhart. Polarization-opponent interneurons in the insect visual system. Nature, 331(6155):435, 1988.

[34] Dimitrios Lambrinos, Hiroshi Kobayashi, Rolf Pfeifer, Marinus Maris, Thomas Labhart, and Rüdiger Wehner. An autonomous agent navigating with a polarized light compass. Adaptive behavior, 6(1):131-161, 1997.

[35] Dimitrios Lambrinos, Ralf Möller, Thomas Labhart, Rolf Pfeifer, and Rüdiger Wehner. A mobile robot employing insect strategies for navigation. Robotics and Autonomous systems, 30(1):39-64, 2000.

[36] Stefano Mafrica, Stéphanie Godiot, Mohsine Menouni, Marc Boyron, Fabien Expert, Raphaël Juston, Nicolas Marchand, Franck Ruffier, and Stéphane Viollet. A bio-inspired analog silicon retina with michaelismenten auto-adaptive pixels sensitive to small and large changes in light. Optics express, 23(5):5614-5635, 2015.

[37] Stefano Mafrica, Alain Servel, and Franck Ruffier. Minimalistic optic flow sensors applied to indoor and outdoor visual guidance and odometry on a car-like robot. Bioinspiration \& biomimetics, 11(6):066007, 2016.

[38] M Mappes and U Homberg. Behavioral analysis of polarization vision in tethered flying locusts. Journal of Comparative Physiology A, 190(1):6168, 2004 .

[39] Richard JD Moore, Karthik Dantu, Geoffrey L Barrows, and Radhika Nagpal. Autonomous mav guidance with a lightweight omnidirectional vision sensor. In 2014 IEEE International Conference on Robotics and Automation (ICRA), pages 3856-3861. IEEE, 2014. 
[40] Richard A Normann and I Perlman. The effects of background illumination on the photoresponses of red and green cones. The Journal of Physiology, 286:491, 1979.

[41] Daniele Palossi, Antonio Loquercio, Francesco Conti, Eric Flamand, Davide Scaramuzza, and Luca Benini. Ultra low power deep-learningpowered autonomous nano drones. arXiv preprint arXiv:1805.01831, 2018.

[42] Matthew M Parsons, Holger G Krapp, and Simon B Laughlin. Sensor fusion in identified visual interneurons. Current Biology, 20(7):624-628, 2010 .

[43] Ramon Pericet-Camara, Michal K Dobrzynski, Raphaël Juston, Stéphane Viollet, Robert Leitel, Hanspeter A Mallot, and Dario Floreano. An artificial elementary eye with optic flow detection and compositional properties. Journal of The Royal Society Interface, 12(109):20150414, 2015.

[44] Thibaut Raharijaona, Lubin Kerhuel, Julien Serres, Frédéric Roubieu, Fabien Expert, Stéphane Viollet, Franck Ruffier, and Nicolas Franceschini. Insect inspired visual motion sensing and flying robots. In Handbook of Biomimetics and Bioinspiration: 2 Electromechanical Systems, pages 565-611. World Scientific, 2014.

[45] Samuel Rossel. Navigation by bees using polarized skylight. Comparative biochemistry and physiology: A: Comparative physiology, 1993.

[46] Samuel Rossel and Rüdiger Wehner. Polarization vision in bees. Nature, 323(6084):128, 1986.

[47] Traci A Sarmiento and Robin R Murphy. Insights on obstacle avoidance for small unmanned aerial systems from a study of flying animal behavior. Robotics and Autonomous Systems, 99:17-29, 2018.

[48] H Schuppe and Roland Hengstenberg. Optical properties of the ocelli of calliphora erythrocephala and their role in the dorsal light response. Journal of Comparative Physiology A, 173(2):143-149, 1993.

[49] Julien R Serres and Franck Ruffier. Optic flow-based collision-free strategies: From insects to robots. Arthropod structure $\&$ development, 46(5):703-717, 2017. 
[50] Wei Shyy, Chang-kwon Kang, Pakpong Chirarattananon, Sridhar Ravi, and Hao Liu. Aerodynamics, sensing and control of insect-scale flappingwing flight. In Proc. R. Soc. A, volume 472, page 20150712. The Royal Society, 2016.

[51] Radwanul H Siddique, Yidenekachew J Donie, Guillaume Gomard, Sisir Yalamanchili, Tsvetelina Merdzhanova, Uli Lemmer, and Hendrik Hölscher. Bioinspired phase-separated disordered nanostructures for thin photovoltaic absorbers. Science Advances, 3(10):e1700232, 2017.

-• Outstanding work on the stucture of a black butterfly to improve the yield of photovoltaic cells.

[52] Young Min Song, Yizhu Xie, Viktor Malyarchuk, Jianliang Xiao, Inhwa Jung, Ki-Joong Choi, Zhuangjian Liu, Hyunsung Park, Chaofeng Lu, Rak-Hwan Kim, et al. Digital cameras with designs inspired by the arthropod eye. Nature, 497(7447):95-99, 2013.

[53] Mandyam V Srinivasan. Visual control of navigation in insects and its relevance for robotics. Current opinion in neurobiology, 21(4):535-543, 2011.

[54] Reuben Strydom, Aymeric Denuelle, and Mandyam V Srinivasan. Bioinspired principles applied to the guidance, navigation and control of uas. Aerospace, 3(3):21, 2016.

[55] Graham K Taylor and Holger G Krapp. Sensory systems and flight stability: what do insects measure and why? Advances in insect physiology, 34:231-316, 2007.

[56] Erik Vanhoutte, Stefano Mafrica, Franck Ruffier, Reinoud J Bootsma, and Julien Serres. Time-of-travel methods for measuring optical flow on board a micro flying robot. Sensors, 17(3):571, 2017.

[57] Stéphane Viollet. Vibrating makes for better seeing: from the fly's micro-eye movements to hyperacute visual sensors. Frontiers in bioengineering and biotechnology, 2, 2014.

[58] Yinlong Wang, Jinkui Chu, Ran Zhang, Lu Wang, and Zhiwen Wang. A novel autonomous real-time position method based on polarized light and geomagnetic field. Scientific reports, 5:9725, 2015. 
[59] Timothy L Warren, Peter T Weir, and Michael H Dickinson. Flying drosophila melanogaster maintain arbitrary but stable headings relative to the angle of polarized light. Journal of Experimental Biology, 221(9):jeb177550, 2018.

[60] Rudiger Wehner. Astronavigation in insects. Annual review of entomology, 29(1):277-298, 1984.

[61] Peter T Weir, Miriam J Henze, Christiane Bleul, Franziska BaumannKlausener, Thomas Labhart, and Michael H Dickinson. Anatomical reconstruction and functional imaging reveal an ordered array of skylight polarization detectors in drosophila. Journal of Neuroscience, 36(19):5397-5404, 2016.

[62] Peng Xu, James Sean Humbert, and Pamela Abshire. Analog VLSI implementation of wide-field integration methods. Journal of Intelligent 65 Robotic Systems, 64(3-4):465-487, 2011.

[63] T. York, S. B. Powell, S. Gao, L. Kahan, T. Charanya, D. Saha, N. W. Roberts, T. W. Cronin, J. Marshall, S. Achilefu, S. P. Lake, B. Raman, and V. Gruev. Bioinspired polarization imaging sensors: From circuits and optics to signal processing algorithms and biomedical applications. Proceedings of the IEEE, 102(10):1450-1469, Oct 2014.

[64] Wei Zhi, Jinkui Chu, Jinshan Li, and Yinlong Wang. A novel attitude determination system aided by polarization sensor. Sensors, 18(1):158, 2018 .

-• First quadrotor fitted with a bio-inspired celestial compass. Such an instrument allows the quadrotor to fly close to electric lines where the magnetic field is disturbed. 